\section{Light Energy and Brightness Sensation}

IN his recent communication ${ }^{1}$. Hopkinson suggests that the relationship between $M$, the magnitude of brightness sensations, and $L$, the corresponding physical luminance, is

$$
M=k L^{0.3}
$$

for dark-adaptation conditions ; and

$$
M=k L
$$

for light-adaptation conditions.

In a paper published in $1953^{2}$ an attempt was made to derive response curves for the eye for various states of adaptation, but because of the difficulty of discussing sensation magnitudes the discussion was limited to the physiological response, $N$, which was thought of as the number of nerve impulses per nerve fibre per unit time. The experimental data upon which the attempt was made consisted mainly of two papers by Craik ${ }^{3,4}$. In these papers the effects of adaptation on luminance discrimination and on apparent brightness were reported, but all the results depended on judgments either of equality of brightness, or of a just noticeable difference in brightness, between two fields. From these results, in the paper eited ${ }^{2}$, a self-consistent pattern of physiological response functions was built up and it was concluded that

$$
N=k L^{1 / 3}
$$

for dark-adaptation conditions ; and

$$
N=k L \boldsymbol{p}
$$

where $p$ is not less than 1 and not more than 3 , for light-adaptation conditions.

The agreement between these results and Hopkinson's is most striking. If both analyses are correct one would conclude that sensation magnitudes are proportional to the number of nerve impulses per nerve fibre per unit time (and not, for example, to the logarithm of that number).

There has for a long time been a tendency to regard the sensation-stimulus relationship in vision as a logarithmic one, and this has arisen mainly from two arguments. First, luminance discrimination results have been integrated and results linear with the log. arithm of the stimulus intensity have been obtained. Secondly, changing all the luminances of a scene by the same percentage (as, for example, by viewing it through a neutral filter) does not affect the appearance of the scene appreciably (providing adequate time is allowed for adaptation), whereas adding the same luminance to each part of the scene (as, for example, when the scene is viewed through haze or fog) does affect the appearance of the scene very markedly (even when full adaptation has taken place). However, luminance discrimination results are entirely concerned with just noticeable differences, and there is evidence ${ }^{2}$ that these are governed by random variations in the stimuli or in their perception and not by any relationship between sensation and stimulus magnitudes. As for the constancy of appearance of a scene when viewed through a neutral filter, this would occur if, on placing the filter in front of the eye, adaptation occurred in such a way that the photochemical absorptions in the retina were unchanged; with filters of moderate density this is almost sure to be the case, while for very dense filters the appearance of the scene does not in fact remain constant; so that here again we see that no deductions can properly be made regarding the sensation-stimulus relationship.

If, then, the logarithmic relationship cannot be properly deduced from experiment, we are at liberty to seek other relationships, and Hopkinson's results are another example of the superiority of the power relationship in this context. So long ago as $1920^{5}$

$$
V=k R^{1 / 2}
$$

was suggested as a good approximation to the relationship between $R$, the reflexion factor of a grey, and its position $V$ along a scale of equal visual steps from black to white. So recently as 1952, this same square-root scale (not a logarithmic scale, which is inferior) was again suggested as a convenient first approximation $^{6}$, and even more recently ${ }^{7}$ a cube-root relationship has been proposed in preference to a logarithmic one.

$$
\begin{gathered}
\text { Research Laboratories, } \\
\text { Kodak, Ltd., } \\
\text { Harrow, Middlesex. }
\end{gathered}
$$

'Hopkinson, R. G., Nature, 178, 1065 (1956).

${ }^{2}$ Hunt, R. W. G., J. Photogr. Sci., 1, 149 (1953).

` Craik, K. J. W., J. Physiol., 92, 406 (1938).

- Craik, K. J. W., Proc. Roy. Soc., B, 128, 232 (1940).

Priest, I. G., Gibson, K. S., and McNicholas, H. J., U.S. Nat. Bur. Stand. Tech. Paper, 167, T 167 (1920).

- Judd, D. B., "Colour in Business, Science, and Industry" (Wiley, New York, 1952).

7 Ladd, J. H., and Pinney, J. E., Proc. Inst. Rad. Eng., 43, 1137 (1955).

I AM encouraged to note that Hunt finds my data on brightness magnitude ${ }^{1}$ in broad agreement with his deductions, from a consideration of the neural responses, about the basic sensation-stimulus relations for the eye; but I would not wish to read too much into this agreement. My communication contained only a brief report of these recent brightnessestimate experiments; but I referred to earlier studies made by a method which involved mental estimates of equal, supra-threshold contrasts, and to the broad general agreement but detailed disagreement between these earlier studies ${ }^{2,3}$ and the later work. A more detailed exposition is given in another paper. The differences between the sensationstimulus relations obtained by one method and by another may be important, both theoretically and also in the practice of lighting engineering. They may arise from different experimental conditions; but I cannot avoid the suspicion that there are real differences between the stimulus-contrast relation and the stimulus-brightness relation. Our training leads us to expect that brightness magnitude can be deduced from contrast magnitude, and vice versa, and thence that we can construct a scale of brightness by adding contrasts, either minimal, as Fechner did, or supra-threshold, as I did in my earlier work, just as we can construct a foot-rule by adding inch-intervals one to the other. To a limited extent we can, and this is why these early brightness scales have been so useful in practice. Sooner or later, the employment of these scales leads us into discrepancies, because 'contrast' does not always seem to be the same thing as 'difference of brightness'. Perhaps there is no reason why it should be.

One simple example may suffice. The contrast in a sunlit snowseape or a bright white cloud can be enhanced by looking through a neutral filter of density $D=0 \cdot 3-0 \cdot 7$. This confirms the existence of a 